\title{
Микроминиатюризация делает рентген подчас незаменимым инструментом обеспечения качества
}

\author{
Рассказывает менеджер по ключевым глобальным клиентам \\ направления электроники и продуктовой линейки FEINFOCUS \\ компании YXLON P. Вага
}

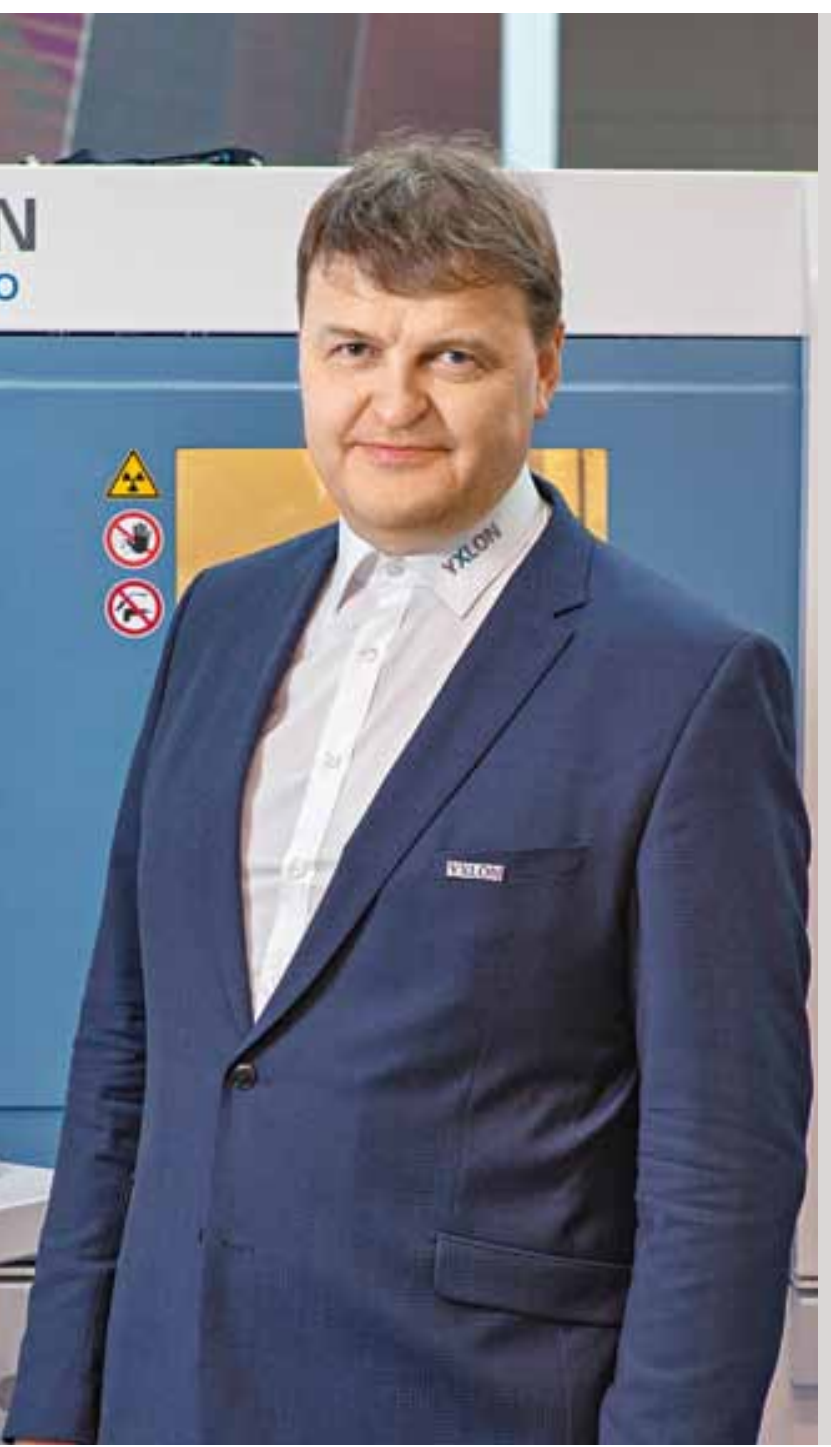

Компания YXLON, которая с 2007 года входит в состав Comet Group, ведущей свою историю с 1948 года, занимает лидирующие позиции в мире в области систем рентгеновской инспекции. Оборудование компании для анализа дефектов электронных изделий работает и на российских предприятиях. На выставке SEMICON China 2019, прошедшей с 20 по 22 марта в Шанхае, компания YXLON представила рынку новые установки, разработанные совместно c Nagoya Electric Works и предназначенные для микроэлектронных производств, в частности для анализа дефектов таких элементов, как переходные отверстия в кремнии (TSV) и медные столбики (copper pillar).

На выставке ElectronTechExpo 2019, состоявшейся в апреле этого года в Москве, менеджер по ключевым глобальным клиентам направления электроники и продуктовой линейки FEINFOCUS компании YXLON Рагнар Вага (Ragnar Vaga) рассказал нам, чем отличаются рентгеновские системы для инспекции электронных и микроэлектронных изделий, в каких еще областях рентген может оказать помощь в разработке и изготовлении электронных устройств, какие решения есть у компании для реализации концепции "Индустрия 4.0", а также поделился своим мнением о перспективах российского рынка.

Господин Вага, ваша компания представляет свое оборудование на выставке ElectronTechExpo не первый год. Как вы оцениваете нынешнее мероприятие в сравнении с предыдущими?

Наверное, я не ошибусь, если скажу, что ExpoElectronica и ElectronTechExро - в своей совокупности единственное крупное мероприятие среди отраслевых выставок российского рынка производства электроники. Поэтому я считаю, что участвовать в ElectronTechExро нужно. Если вас нет здесь, то где вы вообще?

Конечно, об успешности мероприятия для нашей компании можно будет говорить только спустя некоторое время, но, на мой взгляд, выставка проходит 
достаточно хорошо. Если сравнивать с прошлым годом, я не вижу какого-либо шага назад, хотя сказать, что заметен явный рост, я не могу. Что бросается в глаза? В этом году на выставке демонстрируется заметно меньше оборудования. я не увидел здесь ни одной установки рентгеновской инспекции наших уважаемых конкурентов в классе High-end. Для нас это, конечно, хорошо: мы выделяемся на общем фоне, представляя на стенде нашего дистрибьютора в России - компании «Глобал Инжиниринг» - систему YXLON Cheetah EVO. Вообще, я не могу не отметить, что "Глобал Инжиниринг» каждый год подходит к участию в выставке всё более комплексно и профессионально, и мы в этом отношении очень довольны.

В качестве недостатка выставки этого года я отметил бы то, что первый день мероприятия приходится на понедельник. Это неудобно для многих посетителей, приезжающих издалека, и для экспонентов, вынужденных из-за этого проводить подготовительные работы в выходные.

\section{Как вы оцениваете динамику российского рынка} в целом? Можно ли сказать, что рынок в этом году также не лучше, но и не хуже, чем в прошлом?

Для нас этот год в России начался очень хорошо. Я этому даже удивлен. Как правило, начало года бывает довольно спокойным, потому что компании стремятся израсходовать выделенные средства, бюджеты в конце года, после чего наступает затишье

Но в этом году к данному моменту мы уже продали пять систем YXLON Cheetah EVO. Это очень позитивно еще и потому, что раньше в заказах от российских компаний превалировали более компактные системы YXLON Cougar EVO, и то, что в России повышается интерес к установкам Cheetah, указывает на рост ожиданий от систем рентгеновской инспекции у российских заказчиков.

Конечно, на основании такого короткого промежутка времени делать выводы об однозначной тенденции еще рано, но я думаю, что в этом году нас ожидает рост российского рынка рентгеновских систем, пусть и небольшой. Этому будут способствовать общие драйверы в электронике - прежде всего, конечно, непрекращающаяся миниатюризация. Изделия становятся всё меньше и меньше, и для контроля их качества всё чаще не обойтись без рентгена.

Второй важный общемировой драйвер - «Индустрия 4.0". Мы видим, что в России данное направление развивается медленнее, чем в Европе в целом, внедрение соответствующих решений занимает больше времени. Тем не менее здесь этот процесс тоже имеет место, и мы уверены, что он положительно скажется на российском рынке производства электроники. Просто нужно быть терпеливым.

И если говорить о том, как развивается наш бизнес в России, конечно, нельзя обойти вниманием усилия нашего дистрибьютора. Мы начали сотрудничество с ГК "Глобал Инжиниринг» шесть лет назад. Сейчас в их линейку поставок входят решения от таких компаний, как ASM Assembly Systems, Rehm Thermal Systems и т.п., но мы были, пожалуй, первым производителем оборудования класса High-end, который доверился этой тогда еще совсем молодой компании. До этого у нас было несколько дистрибьюторов в России. Я нехочу сказать про них ничего плохого, просто в какой-то момент мы решили, что лучше работать на этом рынке с одним сильным партнером. И я считаю, что мы не ошиблись в выборе. "Глобал Инжиниринг" подходит к своему делу очень профессионально, и это еще одна причина моего ожидания роста нашего бизнеса на российском рынке.

\section{Tо, что в России повышается интерес к установкам Cheetah, указывает на рост ожкиданий от систем рентгеновской инспекции у российских заказчиков}

\section{Какие успехи у вашей компании в глобальном масштабе?}

Рентгеновские системы - лишь одно из целого ряда направлений деятельности Comet Group, в состав которой входит компания YXLON. Среди этих направлений - радиочастотные устройства для контроля плазмы в системах для полупроводниковой и смежных отраслей. Этой областью занимается компания Comet Plasma Control Technologies (Comet PCТ) в составе группы.

Еще одно интересное направление - ebeam. Это достаточно новый и растущий бизнес. Здесь применяется электронно-лучевая технология для различных задач, в том числе для стерилизации. Одним из крупнейших клиентов является Tetra Pak - наверняка, хорошо знакомая вам компания, занимающаяся упаковкой для пищевых продуктов.

Наконец, рентгеновское направление Comet Group само по себе делится на три части. Источники излучения, выпускаемые под брендом СОМЕТ, используются очень широко: не только в наших системах, предназначенных для электронных производств, но и в оборудовании для таких отраслей, как автомобильная, судостроительная, авиационная и т. п. Большинство 
рентгеновских сканеров в аэропортах оборудованы источником СОМЕТ.

Вторая часть рентгеновского направления - это переносные системы, которые применяются, напри мер, для поиска дефектов в крупных объектах, таких

\section{Мы изготавливаем как стандартные}

\section{системы, так и кастомизированные, чем очень гордимся, потому что сейчас у многих заказчиков, особенно крупных, возникает необходимость в нестандартных решениях}

как корабль. Безусловно, такой объект невозможно поместить в камеру стационарной рентгеновской установки, а переносные модули можно внести, допустим, внутрь отсека и произвести необходимые исследования. Также такие переносные системы используются для диагностики трубопроводов, в том числе в удаленных районах.

И наконец, третья часть - это то, чем занимается наша компания: стационарные системы рентгеновского контроля. Я хотел бы подчеркнуть, что мы изготавливаем как стандартные системы, так и кастомизированные, чем очень гордимся, потомучто сейчас у многих заказчиков, особенно крупных, возникает необходимость в нестандартных решениях. Например, иногда требуются установки с очень большими камерами, практически бункерами, внутри которых располагается робот для манипулирования исследуемыми объектами. И такие решения также входят в спектр наших компетенций.

Итак, возвращаясь к вопросу о наших достижениях, чистая выручка в прошлом году на корпоративном уровне Comet Group составила 436,4 млн швейцарских франков, а чистая прибыль - 12,3 млн. В рублях это примерно 27,8 млрд и 782,3 млн соответственно. Согласитесь, достаточно внушительные суммы. Выручка нашего подразделения составила 135,7 млн швейцарских франков или более 8,6 млрд руб. Порядка 30\% от этой суммы приходится на сегмент производства электронных и микроэлектронных изделий, а рост в этом сегменте в прошлом году составил около 7\%. Хотя ближе к концу года наблюдалось некоторое замедление роста, в целом мы очень довольны этим результатом. Его удалось достичь в том числе благодаря тому, что в прошлом году мы уделяли очень большое внимание оптимизации и повышению эффективности процессов - практически на уровне стратегии.
К сожалению, из-за того, что Comet Group представлена на бирже, я не могу сейчас назвать конкретных цифр по началу этого года до публикации официальных результатов, но оценочно для нас год начался в глобальном масштабе очень хорошо, и по объему полученных заказов в первом квартале 2019 года рост по сравнению с тем же периодом 2018 года уже выражается в процентах двузначным числом.

Кроме того, в конце 2018 - начале 2019 года у нас произошли некоторые изменения, с которыми мы связываем большие надежды. В декабре 2018 года в должность вступил новый глава подразделения рентгеновских систем (IXS) Comet Group Томас Венцель (Thomas Wenzel), который с 2015 года был вицепрезидентом по технологиям этого подразделения, а до этого работал в институте Fraunhofer. Также с января 2019 года в YXLON новый вице-президент по глобальным продажам и маркетингу - Барбара Нихтерн (Barbara Nichtern).

У меня нет основанных на независимых исследованиях сведений, какие позиции занимают ведущие компании в области рентгеновской инспекции для производства электроники, но по косвенным признакам с большой степенью вероятности можно сделать вывод, что среди рентгеновских систем типа off-line и at-line для производства электронных устройств, то есть исключая микроэлектронику, мы занимаем лидирующую позицию уже с 2016 года. В области полупроводникового производства нам еще есть, над чем работать. Но и в этом сегменте мы ожидаем хорошего роста. В марте этого года на выставке SEMICON China мы представили рынку новое семейство рентгеновских систем, предназначенных для данной области, которые были разработаны в сотрудничестве с японской компанией Nagoya Electric Works.

В чем заключалась цель сотрудничества с японской компанией? Это связано с особенностями сектора микроэлектроники?

Отчасти, да. На самом деле, это сотрудничество дало положительный эффект для обеих сторон.

В Японии рынок рентгеновских систем достаточно специфический. На нем есть порядка 20-30 компанийпроизводителей разного размера, поэтому сам рынок достаточно конкурентный. Но при этом большинство данных компаний практически не смотрит за рубеж, не выводит свою продукцию на рынки за пределами Японии. Так что, для Nagoya Electric Works сотрудничество с нашей компанией, обладающей не только мощными техническими компетенциями, собственными технологиями в области источников рентгеновского излучения и компьютерной томографии (КТ), но и сильной глобальной сетью продаж, конечно, полезно. 
С другой стороны, Nagoya Electric Works производит очень хорошие системы автоматической инспекции, в особенности для полупроводниковыхпроизводств, где наши компетенции не настолько сильны. Поэтому ихзнания и опыт дополнили наши, и это дало возможность создать новые установки YXLON FF70 CL, FF65 CL и FF65 IL.

Кстати, FFв обозначении установокзначит FEINFOCUS. Эта компания была приобретена Comet Group в 2004 году, а впоследствии данное подразделение было объединено с YXLON. Продолжая использовать этот бренд, мы подчеркиваем, что помним нашу историю.

\section{В чем основное отличие систем для микроэлектро- ники? В разрешении?}

Да, ключевое отличие именно в этом. Например, размер медного столбика может быть всего 25 мкм, а нам нужно непросто увидеть этот столбик, а понять, есть ли в нем пустоты.

У установки FF70 максимальное разрешение составляет 250 нм, что позволяет эффективно исследовать такие элементы микросхем, как TSV, медные столбики и т. п. Установка FF65 обладает несколько меньшим разрешением - до 670 нм, но это также позволяет работать с микроскопическими элементами микросхем с современными типами компоновки.

И второе отличие - это усовершенствованная ламинография. Системы Cheetah и Cougar также способны выполнять ламинографию, но она имеет определенные ограничения, также связанные с разрешением и увеличением. Установки FF70 и FF65, которые специально предназначены для исследования элементов микросхем, снабжены соответствующими средствами автоматической обработки изображений, выявления дефектов, работа которых стала возможной благодаря такому высокому разрешению, которое обеспечивается этими системами.

Чтобы дать более наглядное представление, можно сказать, что когда вы работаете с платой, на которой установлен компонент ВGА, может применяться система Cheetah или Cougar; если вам нужно "заглянуть» внутрь компонента ВGА, уже предпочтительна системa FF65, хотя с рядом таких задач вполне может справиться Cheetah в исполнении с улучшенным детектором и мультифокусной трубкой, но она всё же уступает FF65 в отношении разрешения и возможностей ламинографии; если же вы проводите исследования на уровне кремниевой пластины или кристалла, то необходима установка FF70.

\section{Чем отличается установка FF65 CL от FF65 IL?}

Система FFG5 IL снабжена встроенным загрузчиком для применения на производствах с большими объемами.
Планируется ли дальнейшее совершенствование систем Cheetah и Cougar? Ведь для производителей печатных плат и сборок на них такое разрешение, как у FF70 и FF65, не требуется.

Системы Cheetah и Cougar - очень надежные и хорошо зарекомендовавшие себя. Но мы продолжаем улучшать и их. В частности, мы сейчас работаем над следующей версией системы Cheetah с детектором значительно большего размера. Кроме того, у нас есть планы по применению в этих системах нового запатентованного нами источника рентгеновского излучения. Это должно быть интересным решением. я думаю, что ближе к концу года можно будет говорить об этом более подробно.

Как вы считаете, насколько системы для микроэлектроники могут быть востребованы в России?

Как известно, основной рынок кристального производства сосредоточен в Тайване, Южной Корее, Китае и, конечно, США. Но у нас уже есть потенциальный клиент из микроэлектронной отрас-

КАючевое отличие рентгеновских систем дия микроэлектроники в разрешении: например, размер медного столбика мокет быть всего 25 мкм, а нам нужно не просто увидеть этот столбик, а понять, есть ии в нем пустоты

ли в России, с которым мы начали переговоры еще до появления систем FF70 и FF65 - в прошлом году. Тогда речь шла о системе Cheetah, которая могла бы решать стоящие перед этим клиентом задачи, но она всё-таки не предназначена именно для полупроводниковой отрасли. На этой выставке мы уже рассказали о системах FF70 и FF65 этому клиенту, и он очень положительно оценил новые возможности, которые они открывают. Сейчас мы планируем провести тесты на его изделиях, и я надеюсь, что он останется доволен.

В России сейчас есть определенный интерес к такому направлению, как микросборки на основе низкотемпературной совместно спекаемой керамики - LTCC. Существуют ли особенности у таких изделий с точки зрения применения рентгеновских систем для поиска в них 
дефектов? Готовы ли существующие системы к решению таких задач?

Интерес к таким технологиям, как LTCC, вполне ожидаем, потому что, как я уже говорил, одним из основных драйверов в электронике сейчас является миниатюризация. Производители стремятся уменьшить площадь, занимаемую компонентами и модулями, что заставляет их идти в третье измерение, создавать трехмерные конструкции. Но высота изделий тоже ограничена, и это требует применения новых материалов и технологий, позволяющих изготавливать изделия с большим количеством слоев меньшей толщины, с минимальными размерами межслойных соединений и т. п. И хотя все эти элементы конструкций становятся всё меньше, они должны оставаться достаточно прочными и надежными, чтобы успешно выполнять свои функции в условиях эксплуатации.

В решении этой задачи рентгеновская инспекция, конечно, может быть очень хорошим, а иногда-практически незаменимым помощником. Это касается и технологии LTCC: рентген позволяет выявлять дефекты в межслойных соединениях, пустоты в материалах, отслоения и прочие недостатки в таких изделиях. Конечно, могут быть определенные ограничения, зависящие от структуры, толщины материала. Никогда одно решение не может подходить абсолютно для всех

\section{Ламинография позволяет подробно исследовать иокальные участки,} компоненты, паяные соединения, что обычно представляет наибольший интерес при исследовании таких объектов, как, например, сборки на печатных пnатах

случаев. Но в принципе, микромодули на основе LTCC не являются чем-то уникальным с точки зрения рентгеновской инспекции. Учитывая размеры элементов таких конструкций, они могут быть достаточно хорошей областью применения для рентгеновских систем, которые предназначены для микроэлектроники.

Также поиску дефектов в миниатюрных 3D-конструкциях большую помощь оказывает ламинография.

Чем отличается ламинография от классической КT? В чем ее преимущества и недостатки?

В классической, или полной, томографии образец вращается на 360. Это позволяет получить наиболее полное представление о его геометрии, включая внутренние структуры, потому что в распоряжении исследователя имеются изображения со всех углов обзора.

Ламинография - это тоже вид КТ. Она имеет много названий: томография с ограниченным углом (limited angle tomography), планарная КТ (planar СТ), КТ с наклоном (inclined CТ) и т. п. Суть ее заключается в том, что при получении изображений детектор наклоняется под разными углами в определенных пределах. Затем, в процессе компьютерной обработки, это позволяет построить трехмерную картину, отражающую структуру образца. Но в сравнении с полной КT, объем полученных данных в этом случае меньше из-за ограничения угла наклона детектора, и часть информации приходится восполнять программными средствами. Это, с одной стороны, может приводить к образованию артефактов, а с другой - не позволяет использовать ламинографию для получения изображений при виде сбоку.

Но для определенных задач вид сбоку не нужен. Это относится в том числе и к исследованию печатных плат и структур конструкций микросхем. В этих задачах ламинография не уступает полной КТ. Этому способствует и постоянное совершенствование программ обработки данных для ламинографии.

В то же время, если вы исследуете такой объект, как, например, сборка на печатной плате, вращать его на $360^{\circ}$ бессмысленно. Системе придется "смотреть» на него с расстояния, которое будет определяться шириной платы, несмотря на то, что высота объекта значительно меньше. Поэтому исследование отдельных участков, компонентов, паяных соединений, что обычно представляет наибольший интерес, будет затруднено; сложно будет выявить мелкие дефекты. А ламинография лишена этого недостатка, она позволяет подробно исследовать локальные участки.

Так что ламинография - это не "урезанный" вариант полной КТ, а метод, имеющий свою оптимальную область применения.

Еще один вопрос про области применения рентгена. Для подтверждения качества печатных плат для высоконадежной аппаратуры современные стандарты требуют изготовления и анализа большого количества микрошлифов. Может ли рентгеновская инспекция заменить эту сложную операцию?

Не просто может: это одно из ее назначений. Изготовление микрошлифов - это процесс, занимающий много времени. Кроме того, это разрушающий метод контроля. Даже если вы изготавливаете 
микрошлифы на купонах на технологических полях, то отверстие, которое вы увидите, вы не сможете использовать, и наоборот - с помощью данного подхода вы не сможете изучить отверстие, которое будет фактически работать в изделии. Рентгеновская инспекция, позволяющая исследовать внутренние структуры плат, не разрушая их и не требуя длительных подготовительных работ, - очень хорошая альтернатива микрошлифам.

Но здесь необходимо вновь сделать оговорку: универсальных методов не существует, всегда есть те или иные ограничения. В данном случае исследование структур внутри платы с помощью рентгена может быть затруднено или даже невозможно при сочетании ряда обстоятельств, в частности, если смежные области выполнены из материалов, очень близких по своей структуре и плотности. При этом рентген может оказаться неспособен отличить один материал от другого, и микрошлиф станет практически единственным решением.

Поэтому, я думаю, что полностью от микрошлифов отказаться не удастся, но применение рентгеновских технологий может позволить настолько сократить необходимое их количество, что их изготовление станет скорее исключением, чем правилом.

К этому я хотел бы добавить то, что рентгеновская инспекция позволяет существенно сократить время не только при производственном или приемочном контроле, но и в процессе разработки новых изделий. В качестве примера можно привести то, что у одной ведущей компании - производителя разъемов создание корпуса миниатюрного разъема для поверхностного монтажа требовало 9-10 итераций с изготовлением опытных образцов. Благодаря применению рентгеновской КТ количество итераций удалось сократить до трех.

В сокращении времени разработки новых изделий существенную помощь могут оказать и наши системы горизонтальной КT FF20 СТ и FF35 СТ, у которых есть опция, позволяющая выполнять измерения образцов с метрологической точностью.

В начале интервью вы назвали "Индустрию 4.0" одним из важнейших общемировых драйверов отрасли производства электроники. Каково состояние реализации этой концепции в мире?

я могу долго говорить на эту тему, потому что еще недавно в компании YXLON я занимал должность менеджера по развитию глобального бизнеса, и «Индустрия 4.0» была одним из основных вопросов, которыми я занимался. Но постараюсь быть кратким.

На пути к реализации "Индустрии 4.0" остается много препятствий. Одно из них - большой парк относительно старого, но выполняющего свои функции оборудования на предприятиях нашей отрасли. Это оборудование уже не получится сделать интеллектуальным, а заменять весь этот парк новым, "умным» оборудованием слишком дорого для компаний. Второе ограничение связано с тем, что, как я часто говорю, в «Индустрии 4.0» любая единица оборудования, любая установка - это датчик, который собирает данные об изделиях и процессах. В результате мы получаем огромные объемы данных. Это уже Big Data. И все эти данные нужно обрабатывать. И это только часть проблем.

\section{Рентгеновская инспекция помогает существенно сократить время не только при производственном или приемочном контроле, но и в процессе разработки новых изделий}

Но в направлении «Индустрии 4.0» есть существенные сдвиги. Раньше существовала проблема недоверия между разными компаниями, некоторые из которых являются прямыми конкурентами друг другу, в отношении обмена данными между оборудованием: «Как это так? Чья-то система автоматической инспекции пасты будет говорить моей установке трафаретной печати, что ей делать?» Сейчас уровень доверия растет, компании видят в реализации таких решений общий интерес. Кроме того, стали появляться стандарты: совсем недавно ассоциация IPC выпустила стандарт по интерфейсу CFX - это вертикальный интерфейс для передачи данных от оборудования в информационные системы уровня предприятия. Также появился стандарт Hermes для горизонтального межмашинного взаимодействия, и многие производители оборудования его уже поддерживают. Сейчас работа над дальнейшим совершенствованием стандарта Hermes также перешла под зонтик IPC.

\section{Оборудование YXLON поддерживает стандарт Hermes?}

Наша компания входит в сообщество, работающее над этим стандартом, и мы внимательно следим за данным процессом, но в нашем оборудовании этот интерфейс не поддерживается по одной простой причине. На данном этапе стандарт Hermes предназначен для того, чтобы заменить устаревший интерфейс SMEMA во взаимодействии оборудования в производственной линии. Мы не выпускаем установки типа in-line, то есть встраиваемые 
в линию. Наше оборудование не поддерживало SMEMA, поэтому и заменять нечего. Как только начнется работа над стандартизацией интерфейса для обмена данными с отдельностоящими установ ками, мы включимся в этот процесс уже как активный участник.

Хотя мы специализируемся на оборудовании, работающем вне линии, мы разработали решение, благодаря которому наши системы позволяют скомпенсировать некоторые несовершенства встроенных в линию систем инспекции, при этом обеспечивая высокую оперативность.

Установки автоматической рентгеновской инспекции (АРИ) типа in-line предназначены для того, чтобы достаточно быстро, не задерживая производственный процесс, проверять определенные участки электронных сборок, например паяные соединения компонента BGA, и находить в них дефекты. Однако такие установки имеют ряд ограничений, что

\section{Благодаря решению ProLoop инженер по} качеству может выявяять тенденции и предпринимать корректирующие меры дия повышения выхода годных на основе данных верификации в реккиме, близком к режкиму реального времени

иногда приводит к неоднозначной ситуации. Часто такое случается при подозрении наличия дефекта "голова на подушке", который, как известно, один из самых сложных с точки зрения выявления. Тогда для того, чтобы более точно определить, является ли подозрительное состояние дефектом, и правильно его классифицировать, требуется верификация с помощью установки с бо́льшим разрешением и улучшенными возможностями обработки данных. Здесь и включаются в игру «умные» системы YXLON, которые не только обладают необходимыми для верификации характеристиками получения изображений, но и способны автоматически классифицировать подозрительные состояния.

Еще один пример применения отдельностоящих рентгеновских установок для верификации. Допустим, система трехмерной автоматической оптической инспекции (3D-AOИ) в линии выявляет некоторую непараллельность компонента BGA плате. Само по себе это не дефект. Необходимо проверить, привело ли это состояние к дефектам паяных соединений под корпусом, и это также выполняется на отдельностоящей рентгеновской установке.
Традиционные установки вне линии, так называемые off-line, не имеют с другим оборудованием информационной связи. Поэтому, допустим, при необходимости проверки подозрительного соединения компонента BGA in-line-установка выдает плату инженеру на станцию верификации, он ее загружает в off-line-систему, и начинается поиск этого соединения путем последовательного сканирования по всем выводам компонента. Это занимает время. В результате платы, требующие верификации, начинают накапливаться, и определение дефекта может быть выполнено уже в следующую смену или на следующий день. А ведь всё это время линия продолжает производить изделия, и если эта проблема систематическая, то фактически с линии выходит бракованная продукция.

Чтобы этого избежать, мы предлагаем решение ProLoop, суть которого заключается в том, что наши установки, хотя и не включены в линию, имеют с ней информационную связь. В этом случае мы называем установку не off-line, a at-line, то есть не "вне линии", а "у линии". От системы 3D-АОИ или АРИ в линии установка получает данные о том, где находится подозрительный компонент, паяное соединение и т. п., а также идентификатор платы. Оператору необходимо только положить изделие в установку. По штрихкоду at-line-система идентифицирует плату, считывает реперные знаки и сразу переходит к анализу проблемного участка. Полученные в результате верификации данные могут быть сохранены в самой системе или переданы в общую базу данных. Обработанная информация о дефектах может выдаваться на информационную панель инженера по качеству, и он может достаточно оперативно - конечно, не в режиме реального времени, но близко к этому - выявлять тенденции и предпринимать корректирующие меры для повышения выхода годных.

ProLoop - это решение, которое уже готово к тому, чтобы сыграть свою роль в «Индустрии 4.0». Мы надеемся, что интерфейс для его реализации будет включен во вторую версию стандарта Hermes, и, конечно, будем этому способствовать.

Само собой, здесь не идет речь о том, чтобы решения по дефектам принимались полностью автоматически, без участия инженера. Но я глубоко убежден, что на данном этапе искусственный интеллект еще не готов заменить мозги специалиста, и оптимальным подходом является правильная комбинация автоматизированных средств и человека.

\section{Спасибо за интересный рассказ.}

С Р. Вагой беседовап Ю. Ковалевский 


\section{PBWER M ElEETRONICS}

16-я Международная выставка компонентов и модулей силовой электроники

\section{2-24 октября 2019}

Москва, Крокус Экспо

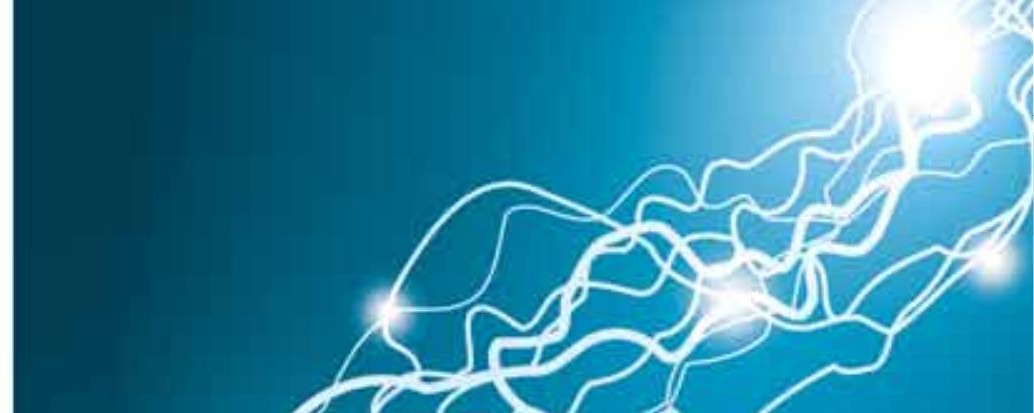

Силовая

suri Approved Электроника

Единственная в России

специализированная

выставка компонентов

и модулей силовой электроники для различных отраслей промышленности

Подробнее о выставке:

$+7(812) 3806009 / 00$ power@mvk.ru 


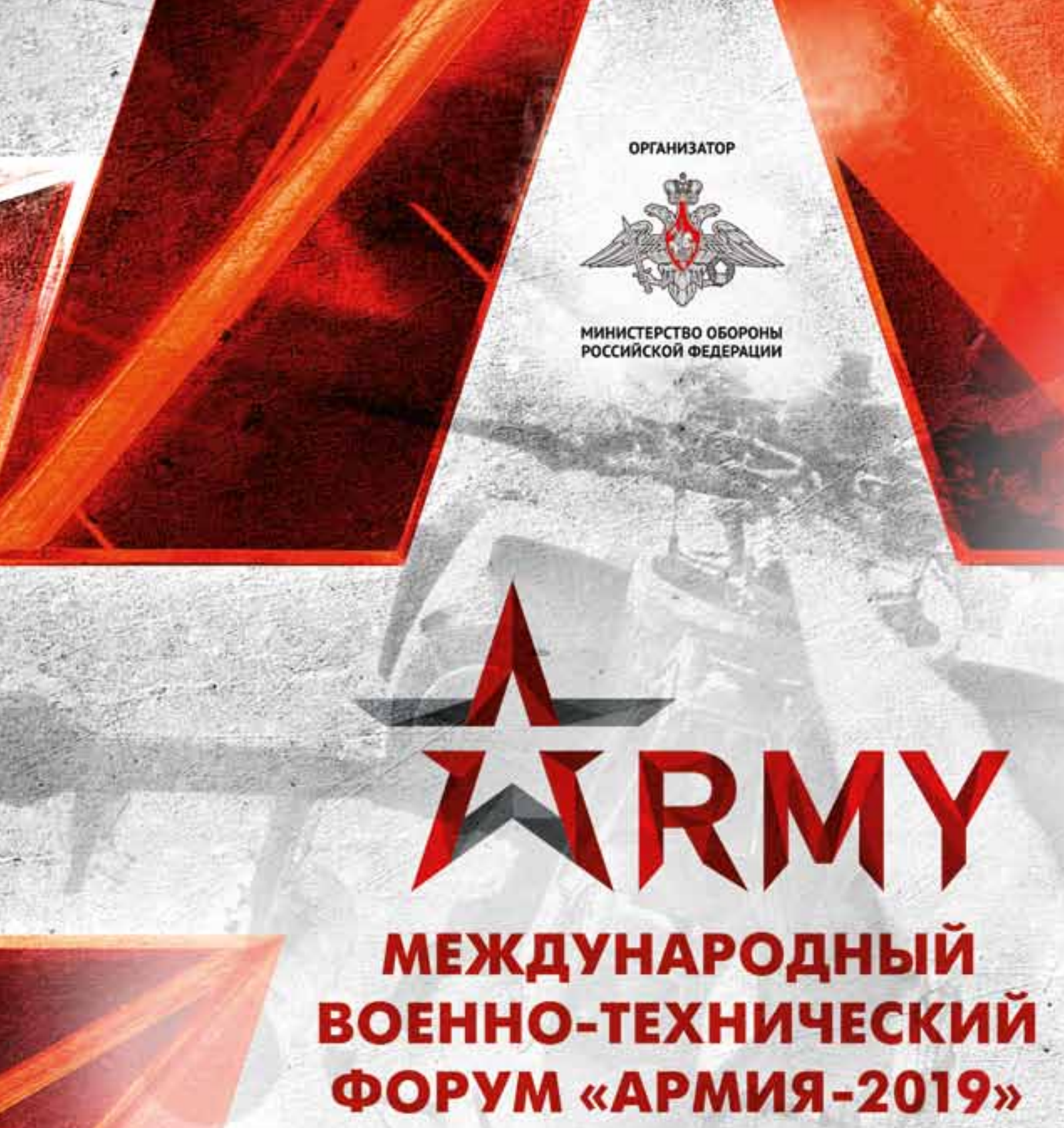

\section{5-30 Июня ПАТРИОТ ЭКСПО}

WWW.RUSARMYEXPO.RU

ВЫСТАВОЧНЫЙ ОПЕРАТОР

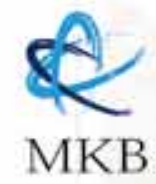

МЕЖДУНАРОДНЫЕ КОНГРЕССЫ И ВЫСТАВКИ 